\title{
La diversidad sexual y sus representaciones en la juventud
}

\section{(1)PSicogente ISSN 0124-0137}

\section{Artículo de Investigación \\ Copyright 2018 by Psicogente \\ Correspondencia de autores: \\ mayra8767@hotmail.com \\ correodejoel@yahoo.com \\ jana-checa@yahoo.com.mx \\ gonzaloovi@gmail.com}

Recibido:03-07-16

Aceptado: 26-04-17

Publicado: 01-01-18

\section{Young people's sexual diversity and its representations}

\author{
Mayra Aracely Chávez Martínez (D), Joel Zapata Salazar (iD), Jana Petrzelová Mazacová ${ }^{(D)}$, \\ Gonzalo Villanueva Ibarra \\ Universidad Autónoma de Coahuila, Ciudad de Coahuila, México
}

Resumen

Objetivo: El objetivo del presente estudio fue indagar la percepción de la diversidad sexual a partir de las representaciones que, a través del discurso, los jóvenes expresan.

Método:Se trabajó con un diseño no experimental, de corte transversal/transaccional de alcance descriptivo. Como instrumento se utilizó la técnica de Redes Semánticas Modificadas; trabajándose los siguientes indicadores: tamaño de la red, peso semántico, diferencia semántica cuantitativa y núcleo de la red. La muestra se integró por 250 jóvenes de ambos sexos, con edades entre los 19 y los 29 años. Se empleó un muestreo no probabilístico, cuyos criterios de inclusión fueron: la disponibilidad y el consentimiento informado.

Resultados: Los resultados demuestran que los sujetos refieren construcciones cada vez menos rígidas en torno a la diversidad sexual, revelando en algunos casos discursos contradictorios en torno a ésta; por un lado, emociones negativas como el miedo y por otro, mayor apertura a la igualdad y al respeto.

Conclusiones: Es necesario considerar que, a pesar de haberse observado una tendencia al cambio en las respuestas de los participantes de este estudio, se debe continuar monitoreando esta tendencia y sus implicaciones en generaciones pasadas y futuras.

Palabras Claves:Diversidad sexual, Jóvenes, Representaciones

Abstract

Objective: The aim of the present study was to investigate the perception of sexual diversity based on the representations that young people express through discourse

Method: We worked with a non-experimental, cross-sectional / transactional cross-sectional design. As instrument was used the technique of Modified Semantic Networks; Working the following indicators: network size, semantic weight, quantitative semantic difference and network core. The sample was composed of 250 young men and women, aged between 19 and 29 years. A non-probabilistic sampling was used, whose inclusion criteria were: availability and informed consent.

Results: The results show that the subjects refer to increasingly rigid constructions around sexual diversity, revealing in some cases contradictory discourses around it; On the one hand, negative emotions such as fear and on the other, greater openness to equality and respect.

Conclusion: It is necessary to con-sider that, despite a tendency to change the responses of the participants of this study, this trend and its implications in past and future generations should be monitored.

Key words:Sexual diversity, Young people, Representations.

Como citar este artículo (APA):

Chávez, M., Zapata, J., Petrzelová, J. \& Villanueva, G. (2018). La diversidad sexual y sus representaciones en la juventud. Psicogente, 21(39), 62-74. http://doi.org/10.17081/psico.21.39.2822 


\section{INTRODUCCIÓN}

La orientación sexual es parte de un proceso de definición del objeto hacia el cual será dirigido el impulso sexual que forma el desarrollo psicosexual, sin que necesariamente se restrinja a este. Según Money y Ehrhardt (1972) el desarrollo psicosexual se considera como un proceso mediante el cual se desarrolla la identidad de género, el rol genérico y la orientación sexual.

La orientación sexo-genérica-sexual indica a la disposición erótica y afectiva a desarrollar actividades sexuales con personas del otro sexo, del mismo sexo o con ambos. Sin embargo, tratar de "etiquetar" a una persona sobre la base de su orientación sexual puede convertirse en un proceso complejo y difícil, pues esta "disposición" no es necesariamente permanente en la vida y puede aplicarse a tres áreas distintas de la sexualidad: el comportamiento sexual, el deseo sexual, y los contenidos de las fantasías sexuales.

Nos podemos referir a Gorguet que explica que la sexualidad: "Es la forma en que cada persona expresa sus deseos, pensamientos, fantasías, actitudes, actividades prácticas y relaciones interpersonales y es el resultado de factores biológicos, psicológicos, socioeconómicos, culturales, éticos, religiosos, espirituales y comunicativos. Existen múltiples expresiones de la sexualidad, tantas como seres humanos" (Gorguet, 2008, p.17).

Relacionado con lo anterior, Foucault propone una "nueva visión de la sexualidad, ya no como una consecuencia de la biología, sino como una construcción social histórica", además de que es esta la que afecta la manera de pensar y entender el cuerpo a través de la historia de nuestros discursos (Mogrojevo, 2008). Dicha "reinvención" del paradigma nos lleva a esclarecer diversos conceptos para que el análisis de los datos obtenidos sea más sólido, ya que el interactuar con condiciones socio-históricas lleva a que los conceptos se tornen más complejos y maleables con el pasar de los años.

\subsection{Situación actual del tema}

El contexto social y político es predominantemente discriminatorio respecto de las personas cuya orientación sexual es diferente a la heterosexual. De acuerdo con datos de la Encuesta Nacional sobre Discriminación en México-ENADIS 2010, realizada por el Consejo Nacional para Prevenir la Discriminación (CONAPRED), la intolerancia hacia los homosexuales presenta una relación inversamente proporcional al nivel de escolaridad, en donde una persona con orientación homosexual no tendría cabida en seis de cada diez hogares de personas sin escolaridad (CONAPRED, 2011). Por otro lado, "nueve de cada diez homosexuales opinan que existe discriminación por sucondición, y 42,8 \% afirma haber sufrido algún acto de discriminación en el último año" (Flores, 2007, p.15).

Durante la mayor parte del siglo XX, el control y disciplina de la sexualidad masculina siguió el camino de la criminalización y la patologización sobre las masculinidades subordinadas. Esta "injusticia cultural" se expresó en la formación de representaciones negativas sobre muchas subjetividades, sobre la diversidad sexual y sobre el género (Fraser, 1997). 
El primer concepto a revisar es la diversidad sexual, cuyo término refiere al hecho de ser diferente y/o desigual, sugiriendo una distancia respecto de "la norma" que es la heterosexualidad. La diversidad implica un continuum de conductas en el que un elemento no tiene valor más fundamental que cualquier otro (Weeks, 2000; Flores, 2007).

Beasley (2006) refiere que el término género se ha usado para indicar que la naturaleza no dice necesariamente mucho acerca de la organización de las identidades y prácticas sexuadas. Un cuerpo masculino no necesariamente resulta en una masculinidad social, en una identidad personal considerada masculina. El género en este escenario es visto como una referencia a la construcción social.

El constructivismo del imaginario social tiene que ver con lo que las personas construyeron juntas, con la reproducción de relaciones sociales y los procesos específicos en lo establecido histórica y culturalmente.

A principios de la década del cuarenta, Linton (1956) realiza un estudio de la diferencia sexual a partir del contraste de estatus, el autor señala cómo todas las personas aprenden a situarse en un estatus sexual específico y cuáles serían las conductas esperadas y socialmente demandadas para dicho estatus. De esta forma, tanto la masculinidad como la feminidad se transforman en identidades psicológicamente constituidas. Como proceso que deviene de la infancia, la mayor parte de las personas coinciden o están de acuerdo tácitamente con el estatus asignado, aunque no siempre sea así. En diversas sociedades, la antropología ha estudiado la existencia de un tercer género: mujeres en algún grado masculinizadas y hombres feminizados en algún grado y se han documentado los hallazgos etnográficamente.

Pero como Lamas (2013) señala, en todos esos planteamientos, subyace una pregunta fundamental que mantiene el debate entre naturaleza/cultura, este es: ¿existe una relación entre las diferencias biológicas y las diferencias socioculturales? Si partimos del supuesto de que los roles sexuales son construcciones culturales e históricas, ¿por qué las mujeres han sido relegadas al ámbito privado y han sido excluidas del poder público?; por el contrario, si los roles sexuales están determinados biológicamente, ¿qué posibilidades existen de modificarlos? El feminismo sintetiza estos planteamientos con un matiz político: ¿por qué la diferencia sexual se traduce en desigualdad social? ¿Por qué las mujeres históricamente han sido el género más relegado?

La diversidad sexual, debido a esa distancia de lo que se considera normal, implica una respuesta de rechazo en algunos grupos sociales que no aceptan esas diferencias. Surge así el fenómeno de la intolerancia, el cual acarrea a su vez conductas de discriminación y agresión hacia la diversidad.

La clasificación popular identifica tres tipos de orientación sexual: la heterosexual (atracción por personas del otro sexo); la homosexual (atracción por personas del mismo sexo), y bisexual (atracción por personas de ambos sexos). En 1942, el biólogo y sociólogo norteamericano Kinsey (citado en Mirabeti-Mullol, 1985) inició una serie de investigaciones sobre sexualidad. Sus estudios lo llevaron a plantear que la orientación sexual es un continuo, que va desde la heterosexualidad exclusiva hasta la homosexualidad exclusiva. 
La homofobia designa el miedo y la aversión irracionales a la homosexualidad y a la comunidad lésbico, gay, bisexual, transexual y transgénero (LGBTT), basados en prejuicios (Agencia de Derechos Fundamentales de la Unión Europea, 2009). Dicho fenómeno se ha visto censurado por diversas instituciones, al menos en el plano público, esto con la intención de que la población general se sensibilice y cambie de actitud paulatinamente. Por otra parte, existen algunas otras más conservadoras como ciertas instituciones eclesiásticas que en lugar de favorecer una conducta más tolerante, estimulan prácticas homofóbicas en sus seguidores.

La homofobia se desarrolla por los mismos mecanismos tanto en la población homosexual como en la heterosexual. No es únicamente un problema que ocurre en las relaciones de los heterosexuales con los homosexuales. Tampoco de estos últimos consigo mismos y con su grupo de referencia, sino que también es un problema de los heterosexuales entre sí y de cada uno para consigo mismo, que puede afectar profundamente a las relaciones con las personas tanto del mismo sexo como del otro sexo (Villanova, Fernández \& García, 1997, p.2).

Dicha estimulación no se ejecuta explícitamente, pero sí se realiza una revisión detenida de su contenido en los discursos que exponen, evidentemente, una intertextualidad que denota una actitud homofóbica de su parte. Se considera que las sociedades homofóbicas tienen un origen social, donde no solamente se encuentra el componente del patriarcado sino también se tiene la fuerte influencia de las instituciones de poder, por ejemplo la Iglesia Católica, que condena a los homosexuales desde la Edad Media, pues de acuerdo a sus creencias "se construye la condena a la sexualidad sin fines reproductivos: desde la masturbación hasta la relación homosexual" (Lamas, 2013; Mercado, 2009).

Hoy en día el cuerpo es un producto social y cultural, donde las prácticas sociales de regulación y dominación de los cuerpos han provocado que exista un desequilibrio notable en la percepción de control sobre nuestro cuerpo y nuestra sexualidad (Campos, 2010).

Foucault nos habla de cómo los temas relacionados con la sexualidad han sido censurados a través de los años creando así un efecto represivo, por lo cual es congruente esperar que los efectos de liberación de dicho poder represivo se manifiesten con lentitud; el hablar libremente del sexo y aceptarlo en su realidad es tan ajeno al hilo de una historia milenaria, es además hostil a los mecanismos intrínsecos a la sexualidad como un tema más en nuestras relaciones cotidianas (Foucault, 1977).

Dentro del marco legal, el matrimonio entre dos personas del mismo sexo se ha abierto camino lentamente en México, donde se empieza a reconocerles el derecho a contraer matrimonio, conformar relación de concubinato e incluso adoptar hijos (Tenorio, 2012).

A raíz de lo anterior se han generado diversos debates donde, a pesar de la lucha porque la preferencia sexual sea reconocida en las instituciones del matrimonio y el concubinato, hay un sector importante de la población que se resiste a aceptar esto aduciendo argumentos de carácter religioso, moral, social y cultural (Tenorio, 2012). 
Actualmente el debate acerca de este tema no se encuentra cerca de llegar a su fin, ya que recae sobre políticas públicas la decisión de que el niño sea adoptado por determinada familia, por lo cual, el proceso cae en la burocracia donde las instituciones de poder conservadoras dominan el manejo de la información, lo que niega la posibilidad de apertura y aceptación hacia el tema.

El respeto a la diversidad identitaria se debe fomentar en todos los espacios sociales y de la vida en el núcleo familiar y en todas sus etapas, desde la infancia hasta la vejez. Debemos inducirnos a una sociedad abierta, tolerante y amistosa, donde no tenga cabida la negación de la diversidad humana (Peixoto Caldas, Fonseca \& Almerida, 2012).

La diversidad afectivo-sexual se debe agregar de forma transversal a lo largo de los diferentes ciclos educativos, asignaturas y materiales de la educación formal para así lograr, la inclusión democrática, de todas las identidades existentes y en todos los ámbitos culturales, económicos, políticos y sociales.

La sociedad mexicana, predominantemente de la religión católica, muy lentamente empieza a aceptar la diversidad sexual, está influida por los prejuicios y pensamientos discriminativos. Se hace un esfuerzo para la transformación de las ideas culturales, sociales y muchas veces hasta políticas para lograr la igualdad y la democracia de una sociedad abierta y libre.

Aceptar plenamente la diversidad sexual requiere la revisión de las clasificaciones que hemos desarrollado sobre la sexualidad y género y también debemos reconocer que no ha sido perfecta.

Como señala Escobar (2007), "La diversidad debe entenderse como un hecho de la sexualidad humana y no puede interpretarse como marginalidad, perversidad o anormalidad. Debe reconocerse la diversidad como derecho a la diferencia, a la ambigüedad y a la singularidad de cada ser humano" (Escobar, 2007, p.92).

Afirman Careaga y Cruz (2004) que hay que "reconocer que las clasificaciones sobre la diversidad sexual no son inamovibles ni definitivas, sino que están en constante movimiento y que se traslapan aún sin darnos cuenta. Incluso, nos reta a mirar un mundo sin categorías, donde las expresiones de la sexualidad, todas, tengan cabida y sean plenamente disfrutadas, un mundo que aún ni siquiera imagino" (p.17).

En México, el reconocimiento legítimo de la existencia de las minorías sexuales, la igualdad con base en la representación y la inclusión en términos de derechos, están lejos de ser una realidad.

Globalmente se puede realizar un diagnóstico y determinar que, en la sociedad mexicana, predominan las prácticas discriminatorias, excluyentes y homofóbicas que son precisamente las que plantean los retos más importantes para la construcción de una sociedad igualitaria e incluyente (Flores, 2007).

La cuestión de la diversidad sexual y de legitimidad de las prácticas o las vivencias homosexuales y bisexuales y de las identidades sexuales y genéricas de las personas travestis y transexuales no se escapa a estas divisiones. De hecho, "es posible afirmar que en el imaginario colectivo mayoritario 
de México y en la visión estatal dichas prácticas e identidades han sido tradicionalmente colocadas dentro de las divisiones de lo insano, inmoral, ilegítimo, ilegal y malo" (Flores, 2007, p.17).

De aquí que el objetivo del presente estudio sea indagar en las configuraciones a partir de las cuales los jóvenes representan a la diversidad sexual a través del discurso; por medio de cogniciones, emociones y prácticas.

\section{MÉTODO}

\subsection{Diseño}

Se trabajó con un diseño no experimental, de corte transversal/transaccional de alcance descriptivo, ya que se busca especificar las propiedades y características del fenómeno de estudio (HernándezSampieri, Fernández \& Baptista, 2014).

En la presente investigación se hizo uso del método fenomenológico; orientándose al acercamiento de la realidad a partir del marco de referencia interna del individuo que se encuentra conformado por todo un conjunto de experiencias, percepciones y recuerdos (Rogers, 1978). Lo anterior nos permite entrar en contacto con la concepción subjetiva que tiene el sujeto acerca de cualquier tema, en este caso, de la diversidad sexual.

\subsection{Participantes}

La muestra seleccionada para el estudio se compuso por jóvenes universitarios del sureste del estado de Coahuila, de las distintas instituciones públicas de educación superior que allí se encuentran. Dicha muestra se integró por 250 jóvenes de ambos sexos, con edades entre los 19 y los 29 años. Se empleó un muestreo no probabilístico, cuyos criterios de inclusión fueron: la disponibilidad y el consentimiento informado. Como condición necesaria, se buscó que los sujetos pertenecieran al mismo colectivo social; para el presente caso, que fueran jóvenes universitarios.

\subsection{Instrumentos}

Dado que se utilizó la técnica de redes semánticas modificadas, se construyó un instrumento conformado por cinco estímulos: diversidad sexual, homofobia, control de la sexualidad, matrimonios del mismo sexo y adopción por parte de parejas del mismo sexo, consignadas cada una en una hoja blanca tamaño media carta. La técnica demanda la utilización de la asociación libre de palabras (Laplanche \& Pontalis, 1983; Tortosa \& Civera, 2006; Mueller, 2003), indicándole al sujeto la siguiente consigna: Lea la palabra estímulo que se encuentra en la parte superior de la hoja y anote las primeras diez palabras que se le vengan a la mente, después se le solicita que les otorgue una jerarquización del 1 al 10, donde 1 es la palabra que más se relaciona con la palabra-estímulo y la 10 la que menos. Finalmente, se le pide que otorgue una carga (positiva, negativa o neutra) a cada palabra con un símbolo de más, de menos o de igual, respectivamente, a cada palabra. 
El instrumento se utilizó en función de su capacidad para posibilitar la construcción de redes semánticas modificadas que detallasen las diferencias individuales y grupales, así como la construcción de un sistema de información de ideas, actitudes, creencias, valores y prácticas vinculadas al objeto de estudio, mismas que, a su vez, facilitan el análisis, la reflexión y la interpretación de la información (Vera, Pimentel \& Batista, 2005).

\subsection{Procedimiento}

La aplicación se realizó de manera grupal, en conjuntos de 40 sujetos. Se les presentó la consigna y se les indicó que si se utilizaban conceptos compuestos, estos no pasaran de dos palabras. La técnica demanda que los participantes permanezcan en silencio durante el proceso, concentrándose en la tarea; así mismo, se les enfatiza la importancia de evitar pensamientos estructurados, reflexivos, lógicos y/o coherentes, durante el procedimiento.

Cada palabra proporcionada por el sujeto, se tipificó como definidora. Con estas se va conformando un universo de asociación o diccionario de asociación; para ello se tomó como punto de partida el registro de la frecuencia de las palabras y el orden primario con que son evocadas. Esto permitió discernir los elementos centrales que concentran el significado de la palabra-estímulo para los sujetos.

Miguélez (1989), al utilizar este método, propone que el investigador ha de realizar ciertas acciones específicas a través de una serie de etapas, que son las siguientes: 1) etapa previa de clarificación de parámetros de los cuales parte el investigador; 2) etapa descriptiva, dentro de la cual se expone una descripción lo más objetivamente posible de la realidad vivida por los sujetos en relación al tema que se pretende investigar; 3) etapa estructural, que implica el estudio y análisis fenomenológico de lo propiamente dicho y 4) debate acerca de los resultados del análisis realizado en contraste con lo planeado con la bibliografía correspondiente.

La información se procesó en una hoja electrónica de cálculo para la construcción de redes semánticas modificadas de acuerdo con la propuesta de Reyes-Lagunes (1993), de quien se retoman los indicadores propuestos para el análisis de resultados: tamaño de la red, peso semántico, diferencia semántica cuantitativa y núcleo de la red.

\section{RESULTADOS}

Para fines de análisis se presentarán principalmente las primeras cinco definidoras con mayor peso semántico, siendo este el más significativo. Las gráficas del núcleo de la red señalan claramente las distancias entre las distintas expresiones de los sujetos participantes. 


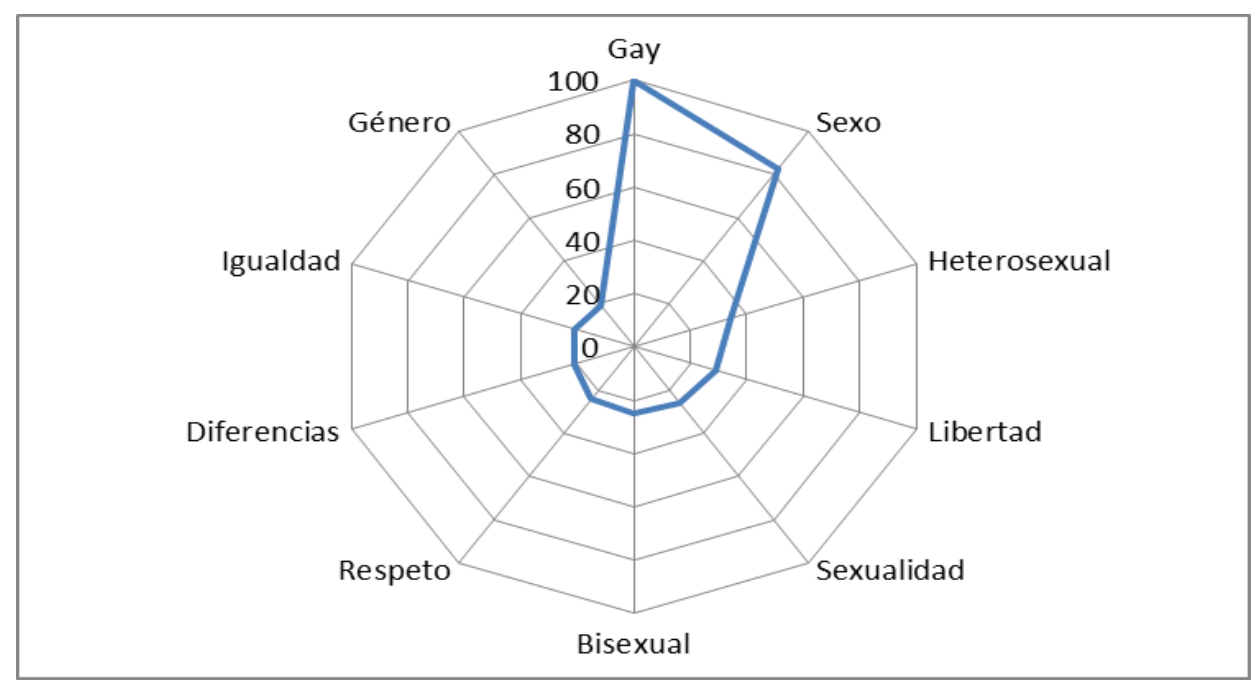

Figura 1.Distancia Semántica Cuantitativa (DSC) Palabra estímulo: Diversidad sexual

En la Figura 1 podemos observar que la palabra con mayor peso semántico es 'gay', en el segundo lugar la palabra 'sexo' y en tercer lugar la palabra 'heterosexual'

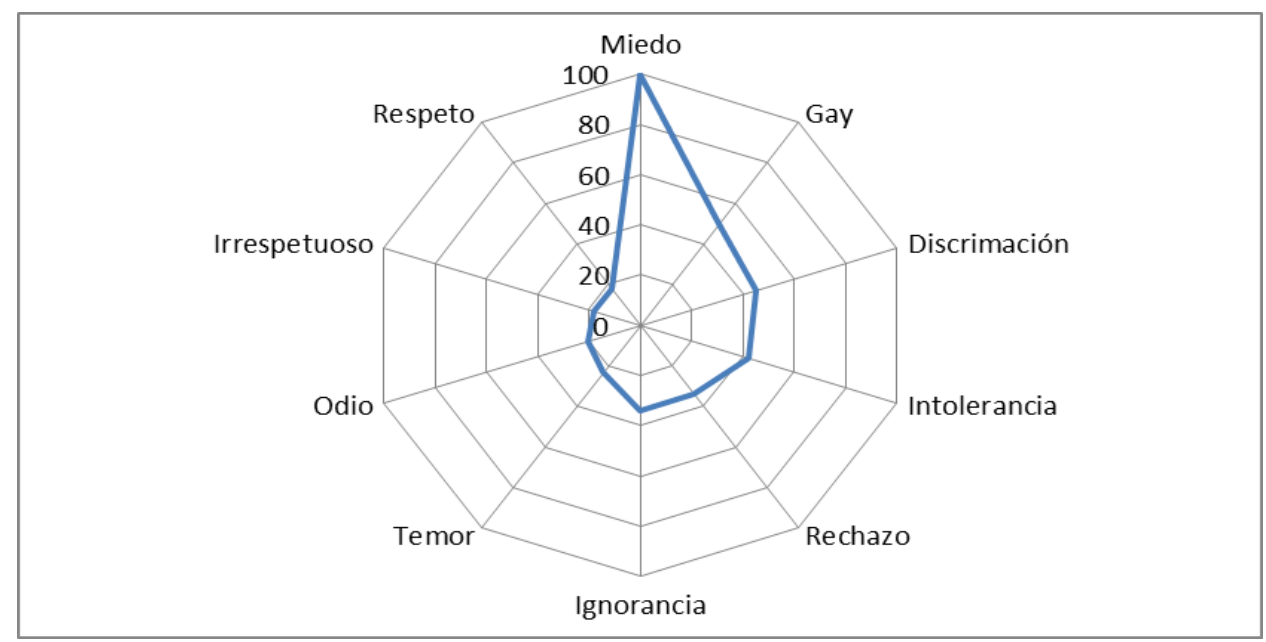

Figura 2.Distancia Semántica Cuantitativa (DSC) Palabra estímulo: Homofobia

En la Figura 2 se detecta que la palabra definidora es el 'miedo', en segundo lugar la palabra 'gay' y en tercero 'discriminación'. 


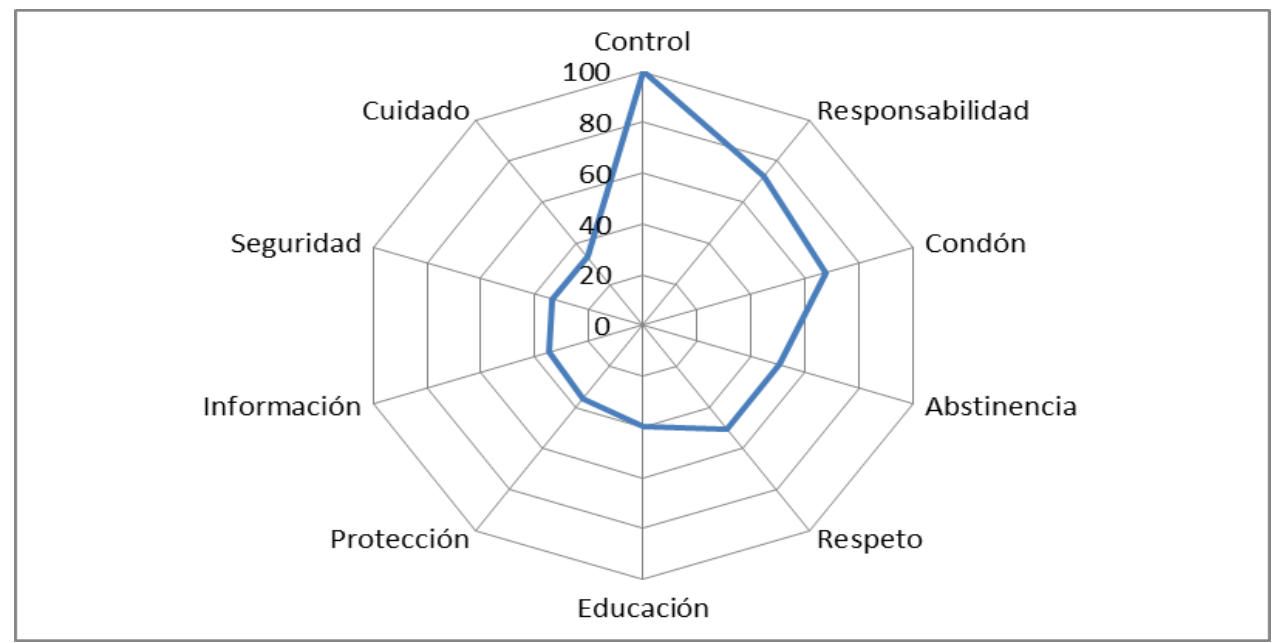

Figura 3. Distancia Semántica Cuantitativa (DSC) Palabra estímulo: Control de la sexualidad

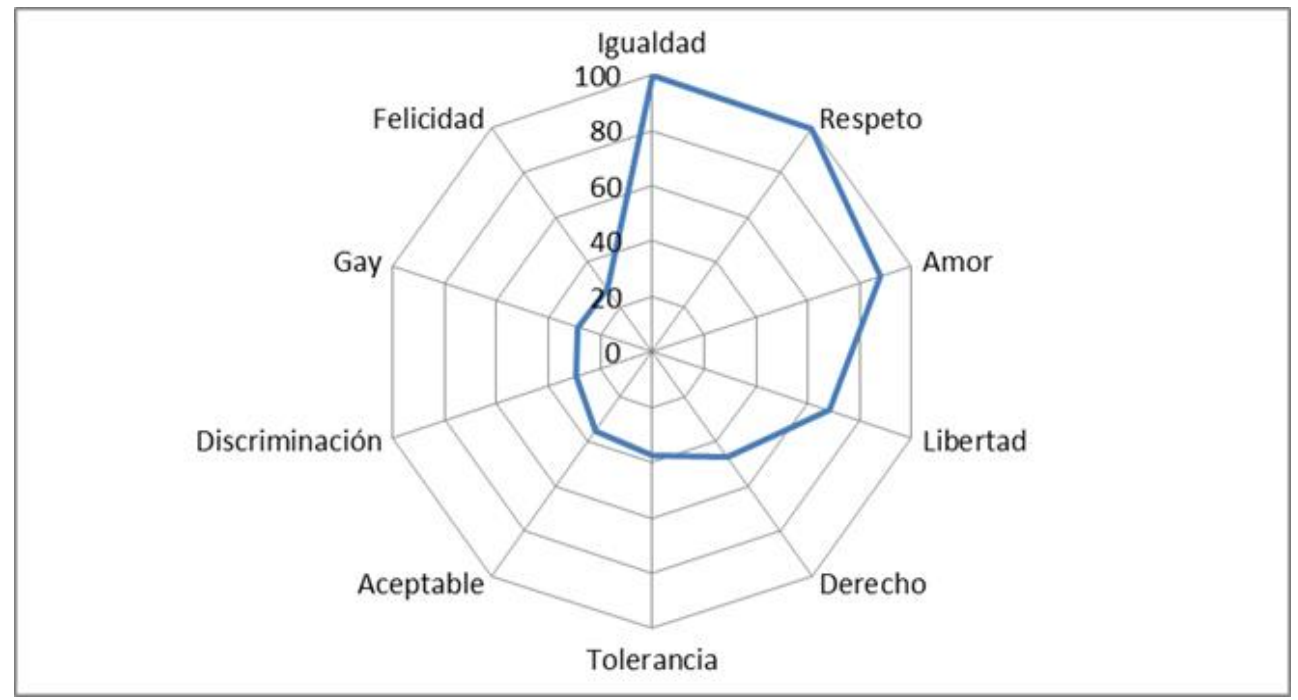

Figura 4.Distancia Semántica Cuantitativa (DSC) Palabra estímulo: Matrimonios del mismo sexo

En la Figura 3 se muestra que la palabra definidora es 'Control' y la segunda 'Responsabilidad'. En la Figura 4 podemos observar que la palabra definidora es la 'Igualdad' y la segunda 'Respeto'. 


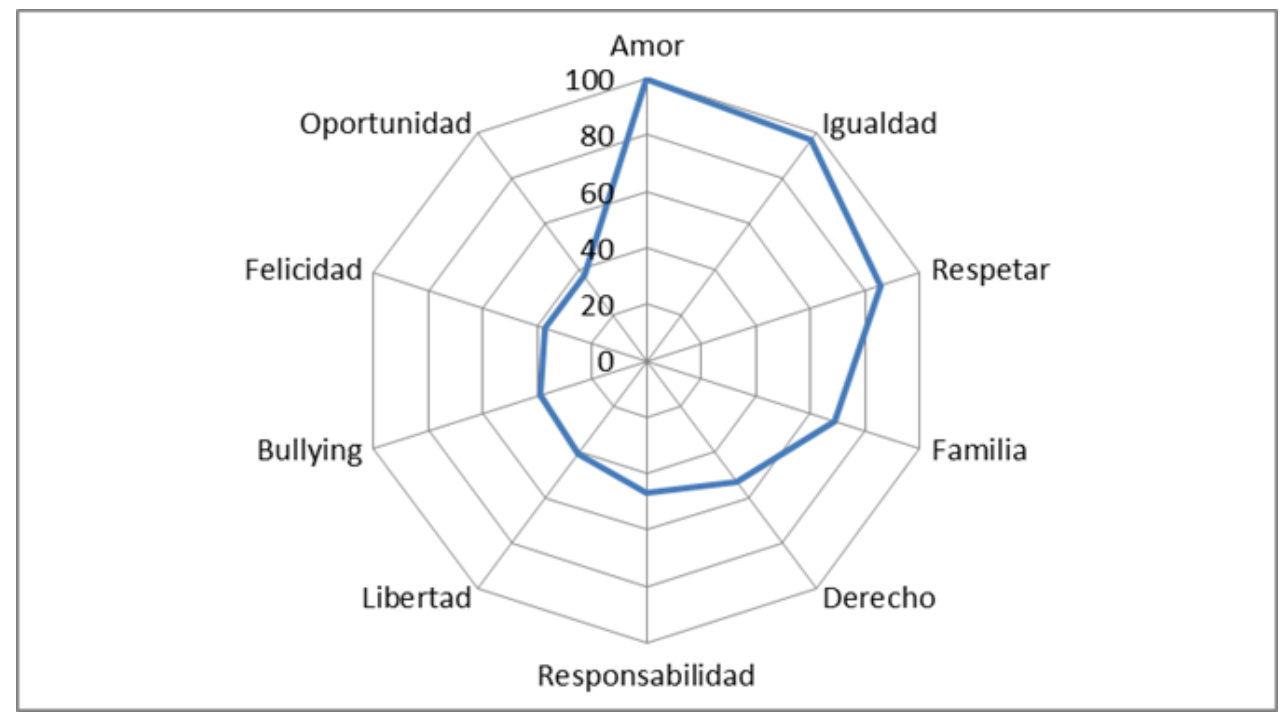

Figura 5.Distancia Semántica Cuantitativa (DSC) Palabra estímulo: Adopción por parte de parejas del mismo sexo

En la Figura 5 podemos observar que la palabra definidora es 'Amor', la segunda 'Igualdad' y la importancia que toma el lugar tercero es 'Respeto'.

\section{DISCUSIÓN}

Dentro de las primeras cinco definidoras el primer concepto estímulo 'Diversidad sexual' encontramos que se relaciona directamente y, de una manera avasalladora, con 'Gay' y un poco por detrás en el peso semántico se encuentra 'Sexo'.

Esto nos habla de una posible desinformación del concepto que se tiene de la diversidad sexual, ya que esta involucra otros aspectos referentes a preferencias sexuales, identidades sexuales, etcétera. Al ser relacionado por los jóvenes con Gay y Sexo, se reduce el espectro de significados que involucra el concepto estímulo. Este es un fenómeno que se debe en parte a la gran cantidad de información a la que los jóvenes se ven sometidos.

Diferentes medios, al sobreinformar, desinforman. Otro aspecto a resaltar es el hecho de la utilización de la palabra 'Gay' en lugar de 'Homosexual'. Esto nos habla de que la sexualidad y la percepción que tienen los jóvenes de la diversidad sexual se ve muy influida por factores sociales, que sobrepasan a los biológicos, la sexualidad se construye de una manera histórica (Mogrojevo, 2008). Dentro de la denominación encontramos matices de discriminación, ya que significados como "cobarde", "afeminado", etcétera, son usualmente asociados con el significado de Gay

La diferencia entre hombres y mujeres en la tolerancia a la homosexualidad del propio sexo es mucho mayor en mujeres que en hombres, posiblemente por las diferentes presiones que se ubican en la construcción de las características del género (Villanova et al., 1997).

En tercer lugar, referente al peso semántico, encontramos a 'Heterosexual'. Aun cuando cuenta con una DSC de 34,01, en contraste con la DSC de 'Sexo' de 82,31, es inesperado observar que una 
palabra definidora sea 'heterosexual' (por delante incluso de 'Bisexual' y la palabra 'Homosexual' no se ve incluida). Su inclusión demuestra la poca tolerancia o adaptación que se tiene a la idea de la diversidad sexual, tornándola rígida y poco flexible; en busca de un ideal para poder ser aceptada, cuando ninguna parte del continuum debe tener más valor que otra (Weeks, 2000; Flores, 2007).

En las dos últimas definidoras, 'Libertad' y 'Sexualidad' encontramos elementos que muestran atisbos de igualdad. La libertad debe ser asociada con la diversidad sexual y, por ende, también a la sexualidad, ya que esta abarca de forma más amplia y holista a las diferentes conductas sexuales de los seres humanos, que se muestran en todos los ámbitos del diario vivir y de la esfera cotidiana de las personas (Gorguet, 2008).

El segundo concepto estímulo es 'Homofobia'. La primera definidora resultante es 'Miedo' con 940 de peso semántico, muy por delante de las que le proceden. Esto nos habla de que se tiene muy en claro la concepción de lo que significa la homofobia, sin embargo, hay diferentes cargas afectivas. Aun cuando se identifique el elemento del miedo, este miedo puede ser un miedo que siente el mismo individuo a lo desconocido, y no necesariamente lo representa por conocimiento de la definición del concepto de homofobia (Agencia de Derechos Fundamentales de la Unión Europea, 2009).

Los estereotipos de género vuelven a hacerse presentes en la segunda definidora, donde se encuentra el término 'Gay', haciendo referencia principalmente a los hombres. Incluso dentro de los grupos subordinados (Homosexuales) por el patriarcado se encuentran grupos doblemente subordinados, que en este caso serían las mujeres lesbianas y las personas transexuales.

Las siguientes dos definidoras cuentan, al igual que la primera definidora, con connotaciones de definición del concepto estímulo, y a la vez con matices que nos hablan de una posibilidad del sentir de los examinados; estas variables son 'Discriminación' e 'Intolerancia'. Sin embargo, la quinta definidora 'Rechazo' es más directa, ya que involucra intrínsecamente una aversión ante el supuesto del que se habla en el concepto estímulo, que es homofobia, que como menciona Lamas (2013) es algo que ha sido sancionado desde la Edad Media.

El tercer concepto estímulo es 'Control de la sexualidad'. La definidora con mayor peso semántico es 'Control' con 635. Esto denota que la mayor parte de losjóvenes universitarios no cuentan con una concepción clara y operacionalizada de lo que involucra el control de la sexualidad y solo conciben su restricción en forma de limitaciones (Cosse, 2012).

La segunda definidora es 'Responsabilidad' con 461 de peso semántico, algo por debajo de "Control". La palabra responsabilidad, por sí misma involucra más matices, es decir, hay una participación activa del sujeto en el proceso del control de la responsabilidad; se analizan diferentes factores como las expectativas a futuro y las consecuencias de los propios actos (Santamaría, 2012).

Las siguientes dos definidoras 'Condón' y 'Abstinencia' hacen referencia al control de la sexualidad en el sentido de control de la natalidad y de enfermedades venéreas. Aun cuando pueden tener el mismo propósito hay una diferencia significativa en su percepción y aplicación. La utilización del condón (aun cuando hay muchos más métodos anticonceptivos, en el caso del control de la 
natalidad) involucra mayor información y conocimiento previo acerca de la sexualidad; congruente con la responsabilidad de la que hablamos anteriormente. La abstinencia, en cambio, aunque cumple los mismos fines, es diferente en el sentido en que elimina o anula en cierta medida las prácticas sexuales, inhibiendo al individuo. Hay que ser cuidadosos al mencionar esto, ya que puede ser motivado por diferentes ideologías que pueden ser tanto personales como influidas externamente en el individuo

Por último, con el mismo peso semántico de 320 que tiene 'Abstinencia' se encuentra 'Respeto'. Los jóvenes universitarios parecen ya empezar a contar con las elaboraciones necesarias para identificar a otro dentro de la sexualidad y no solo como una actividad para conseguir placer propio. Este respeto involucra el reconocimiento de la otra persona en la calidad de ser humano y, por lo tanto, merecedor de consideraciones, de ser aceptado y tomado en cuenta.

El cuarto concepto estímulo es 'Matrimonios del mismo sexo' y sus dos primeras definidoras, con casi idéntico peso semántico de 619 y 615 son 'Igualdad' y 'Respeto' seguidas muy de cerca por 'Amor' y 'Libertad', y por último, 'Derecho'.

La formación de familias de una pareja homosexual es un fenómeno nuevo y, por lo tanto, surgen muchas preguntas sometidas a la nueva construcción del imaginario social que se está erigiendo en la nueva realidad social.

La pronunciación de los grupos LGBT también ha contribuido en este proceso, buscando reivindicar sus derechos sociales y políticos; ganando cada vez más espacios de participación y beneficios legales como el matrimonio, para la sucesión de bienes en caso de una fatalidad (González, 2001).

En este caso se mencionan juntas las definidoras por tener un contenido similar en el sentido de aceptación y de mirada y connotación positiva del concepto estímulo de matrimonios del mismo sexo. No obstante anteriormente se analizaron las primeras 5 definidoras con mayor carga semántica, en este caso es importante señalar que dos de la definidoras finales (la octava, 'Discriminación' y la novena, 'Gay') contienen cargas negativas, de duda o dificultad de aceptación.

Es muy interesante observar cómo la visión de los matrimonios de personas del mismo sexo comienza a ser aceptada dentro de la población juvenil, lo que se podría considerar acorde a las nuevas políticas implementadas a nivel estatal sobre las relaciones formales de personas homosexuales (Tenorio, 2012). Sin embargo, se debe ser precavido al afirmar que son estas políticas de género las causantes de que el marco de tolerancia comience a ampliarse, ya que se puede deber a diferentes factores como los medios de comunicación, la sexualidad más abierta que se vive actualmente o incluso a factores de socialización.

El quinto y último concepto estímulo es 'Adopción por parte de parejas del mismo sexo'. La distribución del peso semántico en este concepto fue bastante parecida dentro de las primeras definidoras, demostrando un amplio abanico de definiciones con las que cuentan los jóvenes. 'Amor', 'Igualdad', 'Respetar', 'Familia' son las primeras cuatro definidoras dentro de la lista.

Al igual que en el concepto estímulo anterior, la mayoría de las definidoras cuentan con una connotación positiva, y nos habla de una aceptación mayor de las adopciones por parte de parejas 
del mismo sexo. La única definidora que pone un poco en juicio lo anterior es la octava, 'Bullying', ya que dentro del imaginario de algunos jóvenes existe el miedo a ser estigmatizados por el hecho de tener padres del mismo sexo.

Es interesante ver cómo la construcción histórica de la vida en pareja evoluciona más allá de algo biológico referido a la reproducción y supervivencia de la especie. Esta nueva concepción es capaz de asimilar e integrar elementos diferentes como una familia compuesta, con niños adoptados y con padres del mismo sexo. Esta maleabilidad que están mostrando las instituciones, como en este caso la familia, son muestra de los cambios de la época posmoderna en la que nos estamos adentrando (Lyotard, 1987).

\section{CONCLUSIONES}

Como principal conclusión se puede mencionar que el discurso de los jóvenes universitarios acerca de la diversidad sexual comienza a ser más variable y dinámico. Esto debido a que comienza a aceptar y a integrar conductas que se escapan de la regla que dicta el patriarcado o la hegemonía dominante.

Esto, como ya lo mencionamos anteriormente, puede ser producto de diferentes factores que van desde los medios de comunicación, la educación escolar, la sensibilización social hasta las políticas implementadas por el Estado.

De una u otra forma, las representaciones de la diversidad sexual comienzan a ser menos rígidas y con mucha más apertura hacia el cambio.

Sin embargo, se debe mencionar que aún se encuentran distintos matices que si bien no se refieren exactamente a una resistencia al cambio, si hay ciertas conductas o ideas que siguen implantadas en el imaginario social, posiblemente como herencia o influencia de generaciones anteriores de jóvenes, que veían o siguen viendo la diversidad sexual como algo indeseable o algo que debe ser escondido, reprimido y castigado.

A pesar de esto, nuestro panorama es bastante prometedor a la hora de hablar de equidad de género. Los jóvenes prometen continuar con esta tendencia al cambio. Lo que se debe seguir observando es cómo evolucionan sus representaciones, qué impacto tienen en generaciones anteriores y qué herencia le dejan a las generaciones venideras.

Nota de Autores

Proyecto de investigación: Diversidad sexual 


\section{MayraAracelyChávezMartínez, Joel Zapata Salazar, Jana Petrzelová Mazacová, GonzaloVillanuevalbarra}

\section{REFERENCIAS}

Agencia de Derechos Fundamentales de la Unión Europea (2009). Homofobia y discriminación por motivos de orientación sexual e identidad de género en los Estados miembros de la Unión Europea. [Versión Adobe Digital Editions]. Disponible

en http://www.movilh.cl/documentacion/2014/Homofobia_y_discriminacion_por_motivos_de_orientacion_se xual_e_identidad_de_genero\%20.pdf

Beasley, Ch. (2006). Gender and Sexuality, Critical Theories, Critical Thinkers. London: Sage.

Campos, E. (2010). Reseña "Historia de la Sexualidad 1: Voluntad del Saber" de Michel Foucault. Sapiens, 11(1), 231233. Dsiponible en http://revistas.urosario.edu.co/index.php/disertaciones/article/view/3848

Careaga, G., \& Cruz, S. (2004). Sexualidades diversas, aproximaciones para su análisis. México: MAP Porrúa.

Consejo Nacional para Prevenir la Discriminación (CONAPRED). (2011). Encuesta Nacional sobre discriminación en México - ENADIS 2010. México: CONAPRED.

Cosse, I. (2012). Sexualidad, adolescentes, amor, placer y control en la Argentina contemporánea. Revista Latinoamericana de población, 6(19), 141-153. Dsiponible en http://www.scielo.br/scielo.php?script=sci_arttext\&pid=S1984-64872010000100010

Escobar, J. (2007). Diversidad sexual y exclusión. Revista Colombiana de Bioética, 2(2), 77-94. Dsiponible en http://www.bioeticaunbosque.edu.co/publicaciones/Revista/Revista4/Articulo3.pdf

Flores, J. (2007). La diversidad sexual y el reto. Dsiponible en http://www.conapred.org.mx/documentos_cedoc/E0005(1).pdf

Foucault, M. (1977). Historìa de la Sexualidad 1. La Voluntad del Saber. Tercera edición. México: Siglo XXI Editores.

Fraser, N. (1997). Iustitia Interrupta. Reflexiones críticas desde la posición "postsocialista". Bogotá: Siglo de Hombres Editores.

González, C. (2001). La identidad gay: una identidad en tensión. Una forma para comprender el mundo de los homosexuales. Desacatos, (6), 97-110. Dsiponible en http://www.scielo.org.mx/scielo.php?pid=S1607050X2001000100005\&script=sci_arttext

Gorguet, I. (2008). Comportamiento sexual humano. Santiago de Cuba, Cuba: Editorial Oriente.

Hernández-Sampieri, R., Fernández, C. \& Baptista, P. (2014). Metodología de la Investigación. México: McGraw-Hill.

Lamas, M. (2013). El género: la construcción cultural de la diferencia sexual. México: Pardo

Porrúa. Laplanche, J., \& Pontalis, J.B. (1983). Diccionario de Psicoanálisis. Barcelona: Labor.

Linton, R. (1956). El estudio del hombre. México: Fondo de Cultura Económica.

Lyotard, J.F. (1987). La condición postmoderna. Madrid: Editions de Minuit.

Mercado, J. (2009). Intolerancia a la diversidad sexual y crímenes por homofobia. Un análisis sociológico. Sociológica, 24(69), 123-156. Dsiponible en http://www.scielo.org.mx/pdf/soc/v24n69/v24n69a7.pdf

Miguélez, M. M. (1989). Comportamiento humano, nuevos métodos de investigación. México: Trillas. 
Mirabeti-Mullol, A. (1985). Homosexualidad hoy. Barcelona: Herder.

Mogrojevo, N. (2008). Diversidad sexual, un concepto problemático. Perspectiva, Revista Trabajo Social, $18,62-71$. Dsiponible en http://www.revistas.unam.mx/index.php/ents/article/view/19577

Money, J. \& Ehrhardt, A. (1972). Man and woman, boy and girl. Baltimore: Johns Hopkins University Press.

Mueller, F.L. (2003). La psicología contemporánea. México: Fondo de Cultura Económica.

Peixoto Caldas, J. M., Fonseca, L. \& Almerida, S. (2012). Escuela y diversidad sexual -¿Qué realidad? Educação em Revista, 28(03), 143-158. Dsiponible en http://www.scielo.br/pdf/edur/v28n3/a07v28n03.pdf

Reyes-Lagunes, I. (1993). Las redes semánticas naturales, su conceptualización y su utilización en la construcción de instrumentos. Revista Mexicana de Psicología y Personalidad, 9(1), 81-97. Dsiponible en http://www.scielo.br/scielo.php?script=sci_nlinks\&ref=000126\&pid=S14137372200700010001900033\&lng= es

Rogers, C. (1978). Terapia, Personalidad y Relaciones Interpersonales. Buenas Aires: Nueva Visión.

Santamaría, A. L. (2012). La responsividad ética. Enclaves del Pensamiento, VI(12), 193-198. Dsiponible en http://www.scielo.org.mx/scielo.php?script=sci_arttext\&pid=S1870-879X2012000200010

Tenorio, L. (2012). Matrimonio entre homosexuales y adopción de hijos. Paradigmas por resolver. Revista de Derecho Privado, 32(2), 311-316. Dsiponible en https://revistas-colaboracion.juridicas.unam.mx/index.php/derechoprivado-ns/article/view/7256/6534

Tortosa, F. \& Civera, C. (2006). Historia de la Psicología. Madrid: McGraw-Hill.

Vera, J. Á., Pimentel, C. E. \& Batista de Albuquerque, J. (2005). Redes semánticas: Aspectos teóricos, técnicos, metodológicos y analíticos. Revista de Sociedad, Cultura y Desarrollo Sustentable, 1(3), 439-451. Dsiponible en http://www.redalyc.org/articulo.oa?id=46110301

Villanova, F., Fernández, L. \& García, I. (1997). Investigación sobre las Actitudes hacia la Homosexualidad en la Población Adolescente Escolarizada de la Comunidad de Madrid. Madrid: COGAM.

Weeks, J. (2000). Sexualidad. México: Paidós.

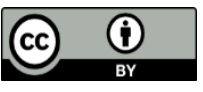

Está obra está bajo: Creative commons attribution 4.0 international license. El beneficiario de la licencia tiene el derecho de copiar, distribuir, exhibir y representar la obra y hacer obras derivadas siempre y cuando reconozca y cite la obra de la forma especificada por el autor o el licenciante 